\title{
AVALIAÇÃO DOS EFEITOS TOXICOLÓGICOS DA IVERMECTINA EM CÃES
}

\author{
Evaluation of Ivermectin Toxicosis in Dogs
}

\author{
Cláudia Turra Pimpão ${ }^{1}$ \\ Rita Maria Venancio Mangrich Rocha ${ }^{1}$ \\ Renata Schaefer ${ }^{2}$ \\ Antônio Felipe Paulino de Figu eiredo Wouk ${ }^{3}$ \\ Silvana Maris Cirio $^{3}$ \\ Evelyn Mara Benato ${ }^{4}$ \\ Luciana Galeb do Amaral Gurgel ${ }^{4}$ \\ Maria Augusta Fronczak ${ }^{4}$
}

\section{Resumo}

A incidência do uso de ivermectina vem aumentando para tratamento de sama sarcóptica em cães, sendo que a dose total utilizada é de $600 \mu \mathrm{g} / \mathrm{kg}$, recomendada por clínicos e vendedores de produtos veterinários. $\mathrm{O}$ objetivo deste trabalho foi avaliar a toxicidade da ivermectina em cães $(600 \mu \mathrm{g} / \mathrm{kg})$. Foram selecionados oito cães adultos SRD, seis foram tratados co m ivermectina $(600 \mu \mathrm{g} / \mathrm{kg})$ divididos em três doses iguais, com intervalos de sete dias, e dois cães mantidos como controles. Mensalmente, foram realizados exames de sangue, de fezes e oftalmoscopias, e após cinco meses da última aplicação, foram realizadas as eutanásias e necropsias dos animais, para exame histopatológico. Os animais experimentados apresentaram parasitas internos, neutrofilia e eosinifilia marcante antes do tratamento. Para os animais tratados com ivermectina, ao exame oftalmoscópico, quatro animais apresentaram deficiência visual, total ou parcial, no exame histopatológico, observou-se hipoplasia linfóide em quatro animais, azoospermia em três deles e um com oligospermia. Todos os animais apresentaram megalocitose hepática de leve à moderada. Houve comprovação da eficiência da ivermectina no controle de parasitas intestinais. A megalocitose hepática observada em todos os animais pode aparecer como conseqüência de insulto tóxico crônico. Houve comprometimento reprodutivo nos machos e as lesões histopatológicas de retina foram compatíveis com os achados macroscópicos.

Palavras-chave: Cães; Ivermectina; Toxicidade.

\section{Abstract}

The incidence of the ivermectin use comes increasing for treatment of scabies in dogs. The aim of this work was to evaluate the toxicity of the ivermectin in dogs $(600 \mu \mathrm{g} / \mathrm{kg})$. Six dogs adults had been selected for treatment with ivermectin $(600 \mu \mathrm{g} / \mathrm{kg})$, divided in three equal doses, with intervals of seven days, and two dogs kept as controls. Monthly, blood tests, coprological techniques and ophthalmoscopy had been carmed through and after five months of the lastapplication were realized the euthanasia and necropsy of the animals, for histopathologic studies. Allanimals had presented intemal parasites, neuthrophilia and eosinophilia before the treatment. For the animals treated with ivermectin, fouranimals had presented total orpartial visual deficiency. Itwas observed azoospermia in three animals, and one with oligospermia. All the animals treated had presented megalocitosis hepatic. It had evidence of the efficiency of the ivermectin in the control of intestinals parasites. There were alterations in male reproductive system and the histopathological injuries of retinal had been compatible with the macroscopy findings.

Keywords: Dogs; Ivermectin; Toxicosis.

1 Médica Veterinária, M.Sc., Pontifícia Universidade Católica do Paraná - PUCPR. Br 376, km 14, Costeira, Cx. Postal 129, CEP 83010-500. São José dos Pinhais, Paraná, Brasil. claudia.pimpao@pucpr.br

2 Médica Veterinária Residente, PUCPR.

3 Médico Veterinário, PUCPR.

4 Médica Veterinária Autônoma. 


\section{Introdução}

No Japão, na década de 1970, foi realizado um experimento que, em termos gerais, baseava-se no isolamento de compostos ativos de um actinomiceto (Streptomyces avermitilis) que acabou por levar à descoberta de uma nova classe de lactonas macrocíclias, as avermectinas (BOOTH; McDONALD, 1992). A ivermectina (dissacarídeo lactona macrocíclico) é formada pela hidrogenação catalítica seletiva das avermectinas $B_{1 \mathrm{a}}$ e $\mathrm{B}_{1 \mathrm{~b}}$ nas proporções respectivas de $80 \%$ ou mais e $20 \%$ ou menos. Seu mecanismo de ação contra os parasitas está na estimulação da liberação do neurotransmissor inibidor GABA (ácido gama-aminobutúrico) na fenda sináptica entre interneurônios do cordão central e neurônios motores (BILL, 1993; McCALL et al., 1996). Assim, a maior liberação de GABA hiperpolariza o potencial de repouso normal das células pós-sinápticas, tomando difícil a neurotransmissão dos estímulos para os músculos periféricos. Portanto, sob a influência de uma pequena quantidade (menos de $1 \mu \mathrm{g} / \mathrm{kg}$, tanto por via oral como parenteral) das avermectinas, os vermes ficam paralisados, sendo posteriormente expelidos pelo organismo (BOOTH; McDONALD, 1992). Como medicamento, a ivermectina foi aprovada, em 1987, pelo Food and Drug Administration (FDA - Estados Unidos) para uso preventivo da dirofilariose canina (Dirofilaria immiti), na dose de $6 \mu \mathrm{g} / \mathrm{kg}$, administrado por via oral, uma vez por mês. Mas a droga, quando administrada em doses maiores, também possui ampla atividade contra, pelo menos alguns estágios de desenvolvimento de nematódeos parasitas e outros artrópodes, bem como é extremamente eficaz contra parasitas intestinais de cães como Ancylostoma caninum, Trichuris vulpis e Toxocara canis. Por via cutânea, na dose $200 \mu \mathrm{g} / \mathrm{kg}$, a ivermectina vem sendo utilizada para tratamento das infestações por sarnas sarcóptica, octodécica e demodécica (BOOTH; McDONALD, 1992; ANDRADE; RODRIGUES, 2002).

Acredita-se que a falta de controle neural dos músculos periféricos mediados pelo GABA em cestódeos e trematódeos torna a ivermectina contra esses ineficaz (GOODMAN; GILMAN, 1995). Estudos demonstraram que a ivermectina possui uma margem de segurança multiplicada por dez em ruminantes, eqüinos e suínos (BOOTH; McDONALD, 1992).
A toxicidade dessa droga em cães da raça Collie já é bem conhecida. Casos isolados de toxicidade em raças como Doberman Pinsher, Old English Sheep Dog e Beagle, utilizando-se doses maiores que as indicadas para bovinos, também foram relatados (CAMACHO; BIAZZONO, 1995; HOPKINS, 1990; PARADIS, 1998a, b). A reação tóxica ocorrida em cães da raça Collie é atribuída a uma característica da barreira hematoencefálica desses animais que permite a passagem da ivermectina em concentrações suficientes para estimular a secreção do GABA, ocasionando depressão do sistema nervoso central (BILL, 1993). As reações adversas da administração da ivermectina são bem documentadas na literatura médico-veterinária. Os sinais de reação tóxica, quando a droga é administrada oralmente, podem ser observados em 24 horas (sendo mais rápido quando administrado por via parenteral) e consistem em: perda do controle motor (incapacidade de manter postura, membros cruzados e ataxia), letargia, fraqueza, perda de reflexos visuais, depressão respiratória, bradicardia, midríase, tremores, hipersalivação, coma e eventualmente a morte (MELO et al., 1995). Importante ressaltar que não há antídoto específico para a intoxicação causada pelo uso desse medicamento (RODER, 1998). Segundo Pimpão et al. (2002), não evidenciaram alterações histopatológicas que pudessem ser diretamente relacionadas a danos provocados pela ivermectina na dose de $600 \mu \mathrm{g} / \mathrm{kg}$ em cães, contudo sugeriram indícios de lesões hepáticas e renais.

Como ocorre uma divergência entre clínicos e patologistas clínicos sobre a toxicidade da ivermectina em cães e a incidência do uso de ivermectina vem aumentando, principalmente no tratamento de sarna sarcóptica, cuja dose recomendada tem sido de $600 \mu \mathrm{g} / \mathrm{kg}$, o objetivo do presente estudo foi avaliar a existência de toxicidade da ivermectina em cães, sem raça definida, decorrente da utilização de dose não estabelecida cientificamente de $600 \mu \mathrm{g} / \mathrm{kg}$ em cães.

\section{Materiais e métodos}

O experimento foi realizado no Laboratório de Farmacologia e Toxicologia Veterinária, do curso de Medicina Veterinária, situado no Câmpus de São José dos Pinhais, na Pontifícia Universidade Católica do Paraná. Foram utilizados 8 cães 
adultos, SRD, sadios, pesando entre 7 e 13 quilos, selecionados aleatoriamente do Canil Municipal de Curitiba. Os cães permaneceram num período de 30 dias, para adaptação, no canil do Câmpus São José dos Pinhais, PUCPR, e alimentados com ração comercial.

Foram realizados exames coprológicos, fundoscópicos e hemograma em todos os animais no início do experimento. Após todos os exames, seis cães foram tratados, por via subcutânea, com três doses de ivermectina ( $200 \mu \mathrm{g} / \mathrm{kg}$ cada), com intervalo de sete dias entre uma aplicação e outra, e dois cães foram tratados com três doses de solução de $\mathrm{NaCl}$ 0,9\%, ou seja, mantidos como grupo controle. Todos os animais foram mantidos nas mesmas condições ambientais.

Durante 5 meses após o último tratamento, foram realizados mensalmente em todos os animais exame coprológico, exame fundoscópico e hemograma. Após este período de acompanhamento, foram realizadas as eutanásias, seguidas por necrópsia e exames histopatológicos. As eutanásias foram realizadas com o uso de thiopental sódico seguido de cloreto de potássio. Os dados laboratoriais obtidos foram avaliados por comparação dos resultados (teste paramétrico).

\section{Resultados e discussão}

De acordo com a Quadro 1, podemos observar os exames coprológicos dos animais experimentados, antes, durante e após três meses de tratamento com ivermectina (600 $\mu \mathrm{g} /$ $\mathrm{kg}$ ) e com $\mathrm{NaCl} 0,9 \%$. Todos os animais apresentaram endoparasitas antes dos tratamentos, sendo que os animais controle continuaram apresentando endoparasitas durante e após o experimento, no entanto, somente dois dos animais tratados com ivermectina voltaram apresentar endoparasitas após 3 meses de tratamento.

\section{QUADR0 01 - Exames coprológicos dos animais tratados com ivermectina (600 $\mu \mathrm{g} / \mathrm{kg})$ e controle (antes, durante e após 3 meses de tratamento).}

Chart 01 - Coprologic examinations of the animals dealt with ivermectina (600 $\mu \mathrm{g} / \mathrm{kg})$ and have controlled (before, during and after 3 months of treatment).

\begin{tabular}{|c|c|c|c|c|}
\hline Coletas & Animais & Antes & Durante & Após 3 meses \\
\hline $\begin{array}{l}\text { Grupo } \\
\text { Ivermectina }\end{array}$ & $\begin{array}{l}\text { CÃO } 1 \\
\text { CÃO } 2 \\
\text { CÃO } 3 \\
\text { CÃO } 4 \\
\text { CÃO } 5 \\
\text { CÃO } 6\end{array}$ & $\begin{array}{l}\text { Ancylostoma caninum } \\
\text { Ancylostoma caninum } \\
\text { Trichuris vulpis } \\
\text { Isoospora canis } \\
\text { Ancylostoma caninum } \\
\text { Trichuris vulpis } \\
\text { Isoospora canis } \\
\text { Trichuris vulpis } \\
\text { Ancylostoma caninum } \\
\text { Trichuris vulpis } \\
\text { Isoospora canis } \\
\text { Ancylostoma caninum } \\
\text { Isoospora canis } \\
\text { Toxocara canis }\end{array}$ & $\begin{array}{l}--- \\
--- \\
--- \\
--- \\
--- \\
---\end{array}$ & $\begin{array}{l}\text { Ancylostoma caninum } \\
\text { Toxocara canis } \\
\text { Ancylostoma caninum } \\
--- \\
--- \\
--- \\
---\end{array}$ \\
\hline $\begin{array}{l}\text { Grupo } \\
\text { Controle }\end{array}$ & $\begin{array}{l}\text { CÃO } 7 \\
\text { CÃO } 8\end{array}$ & $\begin{array}{l}\text { Ancylostoma caninum } \\
\text { Ancylostoma caninum }\end{array}$ & $\begin{array}{l}\text { Ancylostoma caninum } \\
\text { Isoospora canis } \\
\text { Ancylostoma caninum }\end{array}$ & $\begin{array}{l}\text { Ancylostoma caninum } \\
\text { Dipylidium caninum } \\
\text { Ancylostoma caninum }\end{array}$ \\
\hline
\end{tabular}


Os resultados das análises sanguíneas dos animais experimentados antes do tratamento mostraram resposta leucocitária significativa, evoluindo para leucocitose com neutrofilia, algumas vezes com desvio nuclear de neutrófilos à esquerda e com eosinofilia marcante. Os animais tratados com ivermectina apresentaram melhora no hemograma e após o tratamento o hemograma apresentou-se normal. Já os animais pertencentes ao grupo controle continuaram apresentando eosinofilia e neutrofilia durante toda a experimentação.

Ao exame fundoscópico, pode-se verificar, em cinco dos seis animais tratados, graus variáveis de lesões na zona tapetal e pupilar, sendo que estas lesões caracterizam-se por hipe- refletividade tapetal e por sinais de atrofia óptica, com mudança de coloração da pupila. As alterações foram observadas a partir da segunda semana do tratamento e culminaram em 3 indivíduos, ocasionando perda da visão evidenciada por ausência bilateral do reflexo de piscar à ameaça. No exame histopatológico, a retina dos animais tratados apresentou vacuolização da camada de células ganglionares e de fibras nervosas, variando de escassa a moderada, em geral setorial (Figuras 01 e 02). Um dos animais tratados perdeu a visão e foi possível observar a vacuolização por toda a extensão da retina. Os animais controle não apresentaram essas lesões oftálmicas.

\section{FIGURA 1 - Retina de cão adulto, vacuolização da camada ganglionar e de fibras nervosas, Hematoxilina e Eosina. 0bj. 40x}

Figure 1 - Retina of adult dog, vacuolization of the ganglion layer and nervous staple fibers, Hematoxilin and Eosin, Obj. 40x

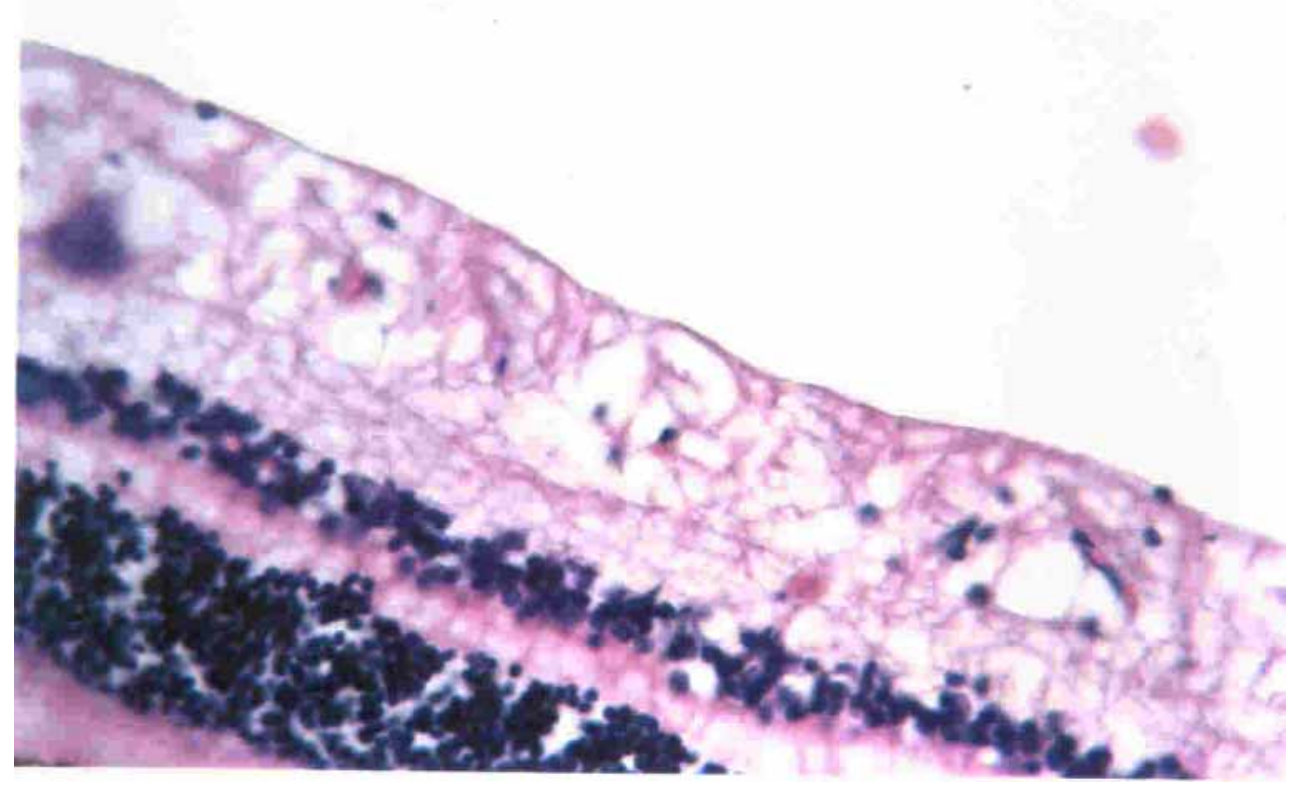




\section{FIGURA 2 - Retina de cão adulto, escassa vacuolização da camada ganglionar e de fibras nervo- sas, Tricrômico de Mallary, 0bj. 40x}

Figure 2 - Retina of adult dog, scarce vacuolization of the ganglion layer and nervous staple fibers, Tricromic of Mallary, Obj. 40x

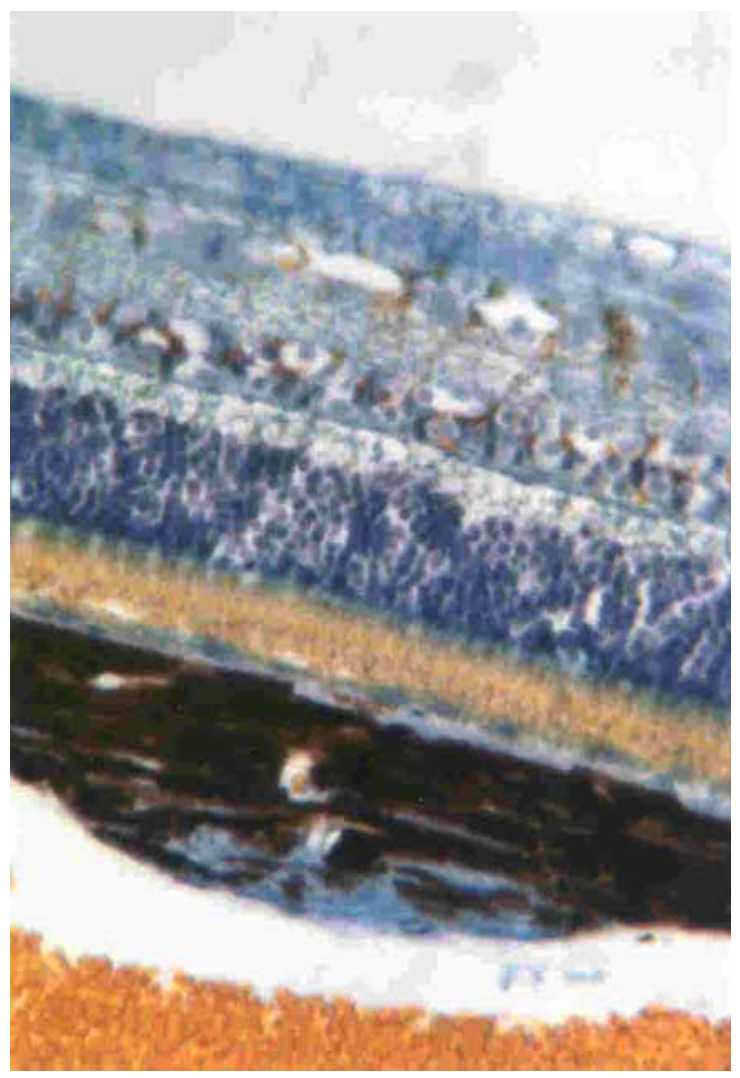

De acordo com os resultados da histopatologia, observamos que quatro animais do grupo tratado com ivermectina apresentaram hipoplasia linfóide, três animais apresentaram azoospermia e um animal apresentou oligospermia, sendo que os animais controle não apresentaram nenhuma dessas alterações. Em todos os animais do grupo tratado com ivermectina, verificou-se megalocitose hepática moderada e nos animais controle megalocitose hepática leve. Nenhum dos animais tratados com ivermectina apresentou quadro de intoxicação aguda.

\section{Conclusões}

A eficiência da ivermectina no controle de endoparasitas neste estudo foi comprovada, conforme Booth; McDonald (1992) e Andrade; Rodrigues (2002). De acordo com Campbell et al. (1983), a ivermectina é ativa contra os dois maio- res filos de parasitas dos animais: os Nemathelminthes (vermes redondos) e os Artropodas (insetos, carrapatos e sarnas).

Clinicamente, à oftalmoscopia, houve lesões de leve à grave, ocorrendo casos de cegueira total. A partir do décimo sexto dia de tratamento com a ivermectina, as alterações encontradas permitem classificá-las como pertencentes ao tipo atrofia retiniana progressiva generalizada e localizada, portanto esses achados reafirmam as observações de Wouk; Kavinski (1985) e Wouk et al. (1985). As lesões macroscópicas da retina neste estudo foram compatíveis com as alterações histopatológicas encontradas.

A megalocitose hepática observada em todos os animais pode aparecer como conseqüência de insulto tóxico crônico, como já havia sido citado por Pimpão et al. (2002).

Houve um comprometimento reprodutivo importante nos machos tratados com a ivermectina neste estudo, também relatado por Sell et 
al. (1996) e Paradis (1998a, b). No entanto, Draurio et al. (1987) não observaram nenhuma alteração na espermatogênese, fertilidade e desempenho reprodutivo.

A ivermectina deve ser usada de maneira criteriosa em cães, evitando usar superdosagens, pois a longo prazo pode aparecer lesões oculares e alterações reprodutivas significativas.

\section{Referências}

ANDRADE, S. F.; RODRIGUES, A. S. Regras básicas para o uso de ivermectina na clínica de pequenos animais. A Hora Veterinária, Porto Alegre, v. 21, n. 125, p. 53-57, 2002.

BILL, R. Pharmacology for veterinary technicians. California: A\&Chnicians, 1993.

BOOTH, N. H.; McDONALD, L. E. Farmacologia e terapêutica veterinária. 7. ed. Rio de Janeiro: Guanabara Koogan, 1992.

CAMACHO, A. A.; BIAZZONO, L. Sinus bradcardia in a Collie dog with ivermectin toxicosis. J. Vet. Res. Anim. Sci. São Paulo, v. 32, n. 4, p. 229-231, 1995.

CAMPBELL, W. C. et al. Ivermectin: a potent new antiparasitic agent. Science, v. 221, p. 823-8, 1983.

DRAURIO, C. P. et al. Reproductive evaluation of male beagles and safety of ivermectin. Am J Vet Res. v.48, p. 1755-1760, 1987.

GOODMAN, J.; GILMAN, X. As bases farmacológicas da terapêutica. 8. ed. Rio de Janeiro: Guanabara Koogan, 1995.

HOPKINS, K.D. et al. Ivermectin toxicosis in a dog. JAm Vet Méd Assoc. v. 197, n. 1, p. 93-94, jul. 1990.

McCAL, J. W.; LINDEMANN, B. A.; PORTER, C. A. Evaluation of ivermectim and milbemycin oxime efficacy against Dirofilaria immitis infections of three and four months' duration in dogs. Am J Vet Res. New Jersey, v. 57, n. 8, p. 1189-1196, ago. 1996.
MELO, M. M.; MARQUES, A. P.; VIANA, F. A. B. Avaliação das enzimas alanina-aminotransferase, aspartato-aminotransferase, fosfatase alacalina e creatinina-fosfoquinase em cães submetidos à administração semanal de ivermectina. Arq Bras Méd Vet Zoot. v. 47, n. 6, p. 753-761, 1995.

PARADIS, M. Ivermectin in small animal dermatology. Part I: pharmacology and toxicology. The Compedium, Montreal, v. 20, n. 2, p. 193-198, fev. 1998a.

PARADIS, M. Ivermectin in small animal dermatology. Part II: extralabel Applications. The Compedium, Montreal, v. 20, n. 4, p. 459-469, fev. 1998b.

PIMPÃO, C. T. et al. Estudo da determinação de doses tóxicas de ivermectina em cães. Rev EstBiol. Curitiba, v. 24, n. 48, p. 39-43, maio 2002.

RODER, J.D. ; STAIR, E.L An overview of ivermectin toxicosis. Am BVetHuman Tox. v. 40, n. 6, p. 369370, dez. 1998.

SELL, E. Heartworm disease: beardie Bulletin, August 1996. Disponível em: <http:// www.beaconforhealth.org/heartworm.htm>. Acesso em: 26 fev. 2002.

WOUK, A. F. et al. Efeitos oculares adversos associados ao uso do ivermectin em cães. In: CONGRESSO BRASILEIRO DE CLÍNICOS VETERINÁRIOSDE PEQUENOSANIMAIS, 8., Porto Alegre, 1985. Anais... Porto Alegre: ANCLIVEPA-RS 1985.

WOUK, A. F.; KAVINSKI, L. C. Caso de cegueira em cão após o uso de ivermectin. In: VIII CONGRESSO BRASILEIRO DE CLÍNICOS VETERINÁRIOS DE PEQUENOS ANIMAIS, 8., Porto Alegre, 1985. Anais... Porto Alegre: ANCLVEPA-RS 1985.

Recebido em: 16/06/2005 Aprovado em: 30/09/2005 